\title{
Exploiting different ways to die
}

\author{
Deirdre A. Nelson and Eileen White ${ }^{1}$
}

Howard Hughes Medical Institute and Center for Advanced Biotechnology and Medicine, Department of Molecular Biology and Biochemistry, Cancer Institute of New Jersey, Rutgers University, Piscataway, New Jersey 08854, USA

\section{Major modes of cell death}

Far from a mere end point, cell death is an essential and highly orchestrated process. Developmental cell death is recognized to play a major role in morphogenesis and tissue sculpting, whereas cell death in mature organisms is essential for tissue homeostasis, wound healing, and elimination of infectious pathogens. Conversely, defective regulation or execution of cell death is also widely recognized as the basis for a spectrum of diseases, including many major human health foes such as diabetes, degenerative disorders, and cancer. Three major types of cell death have been distinguished, all of which contribute to proper development and well-being: apoptosis, autophagy, and necrosis.

Apoptosis is an ordered cell death process in which the entire cell is dismantled within the context of membrane-enclosed vesicles that are recognized and engulfed by phagocytes, thereby preventing release of intracellular components from the dying cells. Studies from many organisms, including Caenorhabditis elegans, Drosophila, and mammals have illustrated that apoptosis is a sophisticated, genetically controlled process integrating a common set of death effectors with a wide range of different stimuli. During development, apoptosis eliminates cells as part of normal morphogenesis, as well as those that incur cellular or genetic damage. In mature organisms, apoptosis ensures proper tissue homeostasis, contributing to normal cell turnover and eliminating both dividing cells with genome damage and cells infected with pathogens, both of which would compromise proper homeostasis. In cells committed to die, proper cell dismantling is carried out by the concerted action of a set of cysteine proteases called caspases. The decision of whether to commit to death is controlled by a large group of regulatory proteins, including the BCL-2 family, inhibitors of apoptosis proteins, and components of death receptor signaling systems. These proteins, in turn, are regulated by the signaling cascades that transduce environmental and cell damage stimuli to the effectors that govern cell fate. The result is preservation of healthy cells, and purging of abnormal, damaged, infected, or unwanted cells in the absence of an immune

\footnotetext{
${ }^{1}$ Corresponding author.
}

E-MAIL ewhite@cabm.rutgers.edu; FAX (732) 235-5795.

Article and publication are at http://www.genesdev.org/cgi/doi/10.1101/ gad.1212404. response (for reviews, see Adams 2003; Danial and Korsmeyer 2004).

Autophagy is also an ordered cellular process involving a large number of gene products whereby cell lysis is prevented, although in this case the cell compartmentalizes and digests itself, as the name implies. As with apoptosis, removal of cells by autophagy may also contribute to proper morphogenesis and tissue sculpting during development, and to tissue homeostasis in mature organisms, although the requirement for autophagy in these processes is not as yet well-defined. In yeast, however, the genetics and physiological role of autophagy have been characterized, in which autophagy plays a major role in adaptation to nutrient limitation by catabolizing intracellular components to ensure survival and in the targeted elimination of specific proteins and damaged organelles. Furthermore, regulation of this pathway by the TOR and PI3 kinases in response to starvation in yeast suggests that autophagy may also be regulated by these signaling cascades in mammals. Thus, autophagy may have complex roles in mammals, functioning both to enable cell survival during starvation or factor deprivation, and to remove damaged organelles or induce death in damaged cells or cells without access to adequate survival factors (for review, see Klionsky and Emr 2000; Levine and Klionsky 2004).

Necrosis refers to a cell that swells and ruptures during its demise, often in response to profound damage or a physical insult, thereby releasing its intracellular components into the surrounding tissue. A major consequence of this is the activation of an inflammatory response. This inflammatory response has tremendous adaptive value for the organism, as it activates both wound healing and an immune response to eliminate or repair the damaged or infected tissue. Although historically considered an unregulated process, more recent data have suggested that necrosis can also occur as a regulated form of cell death. In this regard, necrotic cell death normally accompanies apoptotic cell death in some tissues during development and can be activated in response to several stress stimuli such as inflammatory cytokine signaling and ion channel activation. Necrotic cell death can also occur as an alternative form of death in response to cell damage when apoptosis is blocked (for review, see Proskuryakov and Konoplyannikov 2003). Furthermore, as illustrated by an accompanying report in this issue by Zong et al. (2004), this type of cell death is 
also emerging as an important therapeutic tool, whose induction may play an important role in the treatment of cancer.

\section{Subversion of cell death is cancer's deadly trick}

Cancer is an insidious disease, in which virtually every aspect of cellular control can be subverted to allow the uncontrolled, invasive cellular growth that defeats multicellular cooperation and kills an organism. In testimony to the essential role for proper execution of cell death in tumor suppression, apoptosis is widely recognized as an essential tumor-suppressor system. Indeed, defects in apoptosis are considered a hallmark of cancer, and are known to cooperate with other universal oncogenic lesions during cancer development and progression (Hanahan and Weinberg 2000).

The p53 gene is one of the most frequent targets for mutation in human tumors. p53 has several tumor-suppressive activities, and functions in part to promote apoptosis to suppress tumor formation (Levine et al. 1994). Numerous animal models have corroborated a critical role for p53 in tumor suppression, showing that inactivation of p53 greatly facilitates tumor formation and progression. Although often a direct target for mutation, many p53 regulators are also dysregulated in human cancers, and again animal models have confirmed their role in tumor suppression. As a result, it appears that virtu- ally all clinically relevant human tumors have either mutated p53 itself, or have incurred mutations that disable function of the p53 pathway. In addition to the critical role played by p53 in tumor suppression, p53 also has a profound impact on sensitivity to anticancer cytotoxic agents, such that disabling p53 function during tumorigenesis also reduces the sensitivity of these tumors to such antineoplastic agents, thereby limiting their clinical efficacy (Johnstone et al. 2002).

Another frequent target for mutation in cancers is the BCL-2 family proteins, which regulate apoptotic signaling through the mitochondria. Perhaps the most common form of dysregulation of these proteins results in aberrant up-regulation of antiapoptotic BCL-2 family proteins, although loss of function of proapoptotic BCL-2 family proteins such as Bax and Bak can also occur (Fig. 1). The aberrant survival conferred by these dysregulated proteins prevents cell death in response to a variety of apoptotic stimuli that usually function to prevent tumorigenesis, including DNA damage, hypoxia and nutrient limitation, detachment from extracellular matrix, and escape from toxic cytokines, to name a few. As with the p53-inactivating mutations discussed above, blockade of apoptosis by BCL-2 family proteins also reduces sensitivity to antineoplastic agents that kill cells via apoptosis, thereby limiting their clinical efficacy (Cory et al. 2003; Manion and Hockenbery 2003). Additionally, despite overlap in the tumor-suppressive pathways me-

Figure 1. The role of programmed cell death in tumor development. (Top row) In normal cells, apoptosis eliminates damaged or displaced cells to ensure proper tissue homeostasis. Other growth-limiting stimuli, such as nutrient or essential factor limitation, may trigger autophagy to allow transient survival, but cells will ultimately die by either apoptosis or type II programmed cell death if starvation is prolonged. (Second row) Autophagic-defective cells may have decreased survival in response to nutrient or essential factor limitation, but might also exhibit less efficient cell death in response to normal growth-restricting stimuli, or organelle damage. This could result in excessive cell growth or propagation of damaged cells, which could contribute to tumor formation or progression. (Third row) Apoptotic-resistant cells that fail to die in response to damage or improper environmental survival signals allow for the propagation of damaged or displaced cells, ultimately promoting tumor formation and progression. Such apoptotic lesions can simultaneously impair therapeutic treatments that work by inducing apoptosis (see text). (Bottom row) Tumor cells that depend on aerobic glycolysis to meet energy demands have now been shown to be sensitive to necrotic cell death in response to DNA-alkylating agents, independent of their capacity for apoptosis (see text and accompanying article by Zong et al. 2004). These results provide important insights into an effective class of chemotherapeutic agents, thus facilitating tumor-specific treatment design.

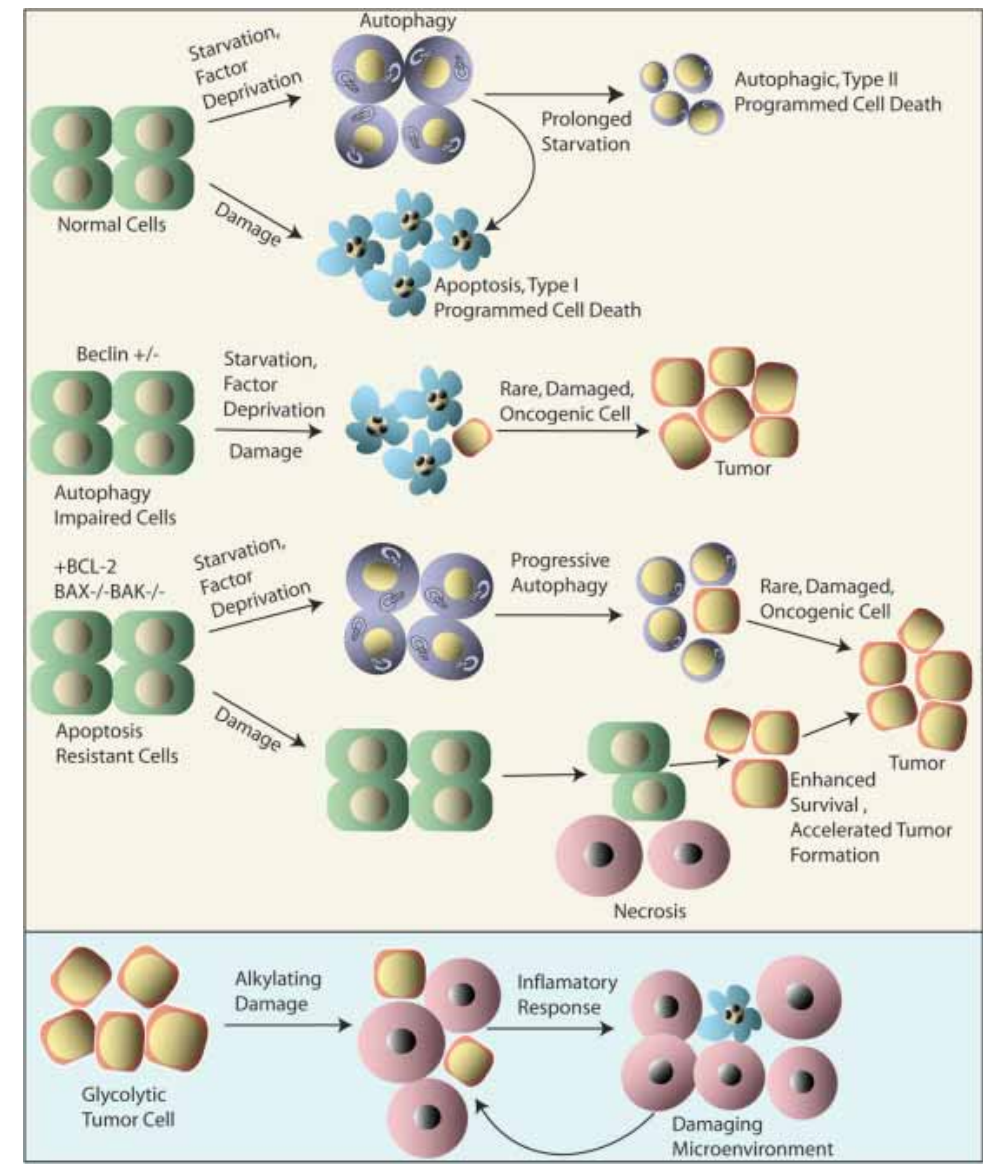


diated by p53 and antagonized by BCL-2, several lines of evidence indicate that mutation of p53 and gain of BCL-2 also have independent functions during tumor progression. First, both p53 mutations and up-regulation of antiapoptotic BCL-2 family proteins are common to many clinically relevant human tumors. Second, both loss of p53 function and abrogation of mitochondrial apoptosis synergize, or are required, in mouse models for solid tumor progression (Hager and Hanahan 1999; Bruckheimer et al. 2000; Degenhardt et al. 2002). Thus, the p53 pathway and BCL-2 family proteins represent distinct targets for inactivation during tumorigenesis, and for anticancer therapeutic intervention.

Many other types of mutations common to tumor formation and progression provide some type of protection from apoptosis, such as activating mutations in RAS, or up-regulation of growth factor receptor tyrosine kinases such as HER2. Although diverse in mechanism, all highlight the central role for apoptosis in tumor suppression. In contrast, the contributions of other cell death modes to tumor suppression, or progression, are considerably less clear.

Recently, however, several lines of evidence have linked defective control of autophagy to tumor development. First, expression of beclin 1, the mammalian ortholog of the yeast autophagy gene Apg6 that is monoallelically deleted in many human tumors, promotes autophagy and inhibits tumor formation in xenografts (Liang et al. 1999). Further, beclin 1 loss correlates with reduced autophagy, and beclin 1 is haploinsuficient for tumor formation in mice, suggesting that a reduction in autophagy can promote tumor formation (Qu et al. 2003; Yue et al. 2003). Second, in an in vitro acini formation system in which autophagy cooperates with apoptosis to create normal acini, interference with either apoptosis or autophagy is sufficient to cause luminal filling that is reminiscent of the hyperproliferative loss of normal tissue architecture that accompanies the early development of human breast cancer (Debnath et al. 2002; Mills et al. 2004). Third, blocking a PDGF autocrine survival loop in malignant glioma cells results in decreased survival with increased autophagy rather than apoptosis, suggesting that autophagy may function normally to eliminate cells without proper access to survival factors (Takeuchi et al. 2004). Thus, impaired autophagy may perturb a death and/or repair mechanism to allow the emergence of oncogenic cells (Fig. 1). Although provocative, for these latter studies, definitive evidence of a role for autophagy in tumor suppression is not available. Thus, whether autophagy, like apoptosis, will emerge as a major mechanism for tumor suppression awaits further clarification.

As for necrosis, it is not clear at present that this form of cell death plays a major role in tumor suppression. On the one hand, tumor cell necrosis can provoke an inflammatory response, thereby stimulating immune surveillance (Fig. 1). On the other hand, chronic inflammation is thought to promote tumor formation and progression, which is the basis for current efforts to use nonsteroidal anti-inflammatory agents for chemoprevention (Fig. 1).
Thus, one consequence of blocking cell death by apoptosis and/or autophagy may be to switch the death of damaged cells to necrosis. This response may be substantially less efficient, thereby decreasing overall cell death. Additionally, necrosis could create a damaging microenvironment and provoke an inflammatory response. This response might function initially to suppress tumor formation, but over time a persistent damaging microenvironment would facilitate mutation, and select for growth-enhancing mutations, thereby promoting tumor progression. Such a mechanism could obviously synergize with more direct mechanisms whereby aberrant survival of damaged cells that are not removed by apoptosis, autophagy, or necrosis could result in propagation of damaged cells and contribute directly to cancer progression.

\section{Turning the trick around: exploiting cell death in cancer control}

As defects in apoptosis are likely universal lesions in allowing tumor formation, restoration or activation of apoptosis in tumors is an active area of anticancer research. In fact, preclinical trials have validated the antitumor efficacy of this approach, and clinical trials are underway for many apoptosis-activating strategies. Conversely, as apoptosis involves many regulators and effectors, this diversity can hamper determination of effective targets for individual tumors, and can contribute to drug resistance during treatment. These obstacles have led to the realization that effective cancer treatment strategies will likely require combinatorial approaches that, together with individual tumor typing and matching with type-specific treatments, will allow the goal of effective tumor-specific therapeutics, or personalized medicine.

An article in this issue of Genes \& Development by Zong et al. (2004) makes substantial contributions to two avenues of this goal. Using genetically defined cells, these investigators show that DNA-alkylating agents cause necrotic cell death, which is equally effective in cells with and without apoptotic defects, and does not require p53 or Bax and Bak (Fig. 1). These results highlight the importance of inducing necrotic cell death for effective therapeutics involving DNA-alkylating agents. These agents then join a growing list of possible anticancer therapeutics that can kill by inducing necrosis, such as cisplatin (Gonzalez et al. 2001) and other cytotoxic agents (Proskuryakov and Konoplyannikov 2003), as well as hypoxic or anoxic region activated drugs such as $\beta$-lapachone (Pardee et al. 2002), which may be important for effective therapeutic treatment of tumors containing cells with apoptotic defects. Furthermore, as many solid tumors and their metastases are heterogeneous at the cellular level, these agents may be required as part of combination regimens needed to eradicate the mixed cell populations that comprise these tumors.

Second, Zong et al. (2004) identify the molecular signature of cells that sensitizes them to necrotic death in response to DNA-alkylating agents. They demonstrate that necrosis in response to DNA-alkylating agents re- 
quires poly(ADP-ribose) polymerase (PARP). Consistent with previous reports in which excessive PARP activity was shown to deplete $\beta$-nicotinamide adenine dinucleotide (NAD) and contribute to cell death in response to excitotoxic stimuli or reperfusion injury, the excessive PARP activity caused depletion of NAD and ATP. They then go on to elucidate another key aspect of cells that permits sensitization to alkylating agents: the metabolic state of the cell. Although required, PARP is not sufficient to sensitize cells to necrosis. Rather, only cells using aerobic glycolysis, as is typical of many cancer cells, are sensitized for killing, and cells using mitochondrial substrates for oxidative phosphorylation are not. This defines a key characteristic of cancer cells, namely, their propensity for aerobic glycolysis, as the key to the tumor cell-specific cytotoxicity of DNA-alkylating agents. These observations are the types of essential links between tumor cell phenotype and sensitivity to chemotherapeutic agent that are essential to allow tailoring of individual tumors to the treatment paradigm to which they will respond best. Of course, realizing personalized medicine will require an integrated array of information relating tumor cell phenotype to effective treatment agents, and these observations move us ever closer to the goal.

\section{References}

Adams, J.M. 2003. Ways of dying: Multiple pathways to apoptosis. Genes \& Dev. 17: 2481-2495.

Bruckheimer, E.M., Brisbay, S., Johnson, D.J., Gingrich, J.R., Greenberg, N., and McDonnell, T.J. 2000. Bcl-2 accelerates multistep prostate carcinogenesis in vivo. Oncogene 19: 5251-5258.

Cory, S., Huang, D.C., and Adams, J.M. 2003. The Bcl-2 family: Roles in cell survival and oncogenesis. Oncogene 22: 85908607.

Danial, N.N. and Korsmeyer, S.J. 2004. Cell death: Critical control points. Cell 116: 205-219.

Debnath, J., Mills, K.R., Collins, N.L., Reginato, M.J., Muthuswamy, S.K., and Brugge, J.S. 2002. The role of apoptosis in creating and maintaining luminal space within normal nd oncogene-expressing mammary acini. Cell 111: 29-40.

Degenhardt, K., Chen, G., Lindsten, T., and White, E. 2002. Bax and Bak mediate p53-independent suppression of tumorigenesis. Cancer Cell 2: 193-203.

Gonzalez, V.M., Fuertes, M.A., Alonso, C., and Perez, J.M. 2001. Is cisplatin-induced cell death always produced by apoptosis? Mol. Pharmacol. 59: 657-663.

Hager, J.H. and Hanahan, D. 1999. Tumor cells utilize multiple pathways to down-modulate apoptosis. Lessons from a mouse model of islet cell carcinogenesis. Ann. NY Acad. Sci. 887: 150-163.

Hanahan, D. and Weinberg, R.A. 2000. The hallmarks of cancer. Cell 100: $57-70$.

Johnstone, R.W., Ruefli, A., and Lowe, S.W. 2002. Apoptosis: A link between cancer genetics and chemotherapy. Cell 108: 153-164.

Klionsky, D.J. and Emr, S.D. 2000. Autophagy as a regulated pathway of cellular degradation. Science 290: 1717-1721.

Levine, B. and Klionsky, D.J. 2004. Development by self-digestion; molecular mechanisms and biological functions of autophagy. Dev. Cell 6: 463-477.

Levine, A.J., Perry, M.E., Chang, A., Silver, A., Dittmer, D., Wu,
M., and Welsh, D. 1994. The 1993 Walter Hubert lecture: The role of the p53 tumour-suppressor gene in tumorigenesis. Br. J. Cancer 69: 409-416.

Liang, X.H., Jackson, S., Seaman, M., Brown, K., Kempkes, B., Hibshoosh, H., and Levine, B. 1999. Induction of autophagy and inhibition of tumorigenesis by beclin 1. Nature 402: 672-676.

Manion, M.K. and Hockenbery, D.M. 2003. Targeting BCL-2related proteins in cancer therapy. Cancer Biol. Ther. 2: S105-S114.

Mills, K.R., Reginato, M., Debnath, J., Queenan, B., and Brugge, J.S. 2004. Tumor necrosis factor-related apoptosis-inducing ligand (TRAIL) is required for induction of autophagy during lumen formation in vitro. Proc. Natl. Acad. Sci. 101: 34383443.

Pardee, A.B., Li, Y.Z., and Li, C.J. 2002. Cancer therapy with ß-lapachone. Curr. Cancer Drug Targets 227: 242.

Proskuryakov, S.Y. and Konoplyannikov, A.G. 2003. Necrosis: A specific form of programmed cell death? Exp. Cell Res. 283: 1-16.

Qu, X., Yu, J., Bhagat, G., Furuya, N., Hibshoosh, H., Troxel, A., Rosen, J., Eskelinen, E.L., Mizushima, N., Ohsumi, Y., et al. 2003. Promotion of tumorigenesis by heterozygous disruption of the beclin 1 autophagy gene. J. Clin. Invest. 112: 1809-1820.

Takeuchi, H., Kanzawa, T., Kondo, Y., and Kondo, S. 2004. Inhibition of platelet-derived growth factor signalling induces autophagy in malignant glioma cells. Br. J. Cancer 90: 10691075.

Yue, Z., Jin, S., Yang, C., Levine, A.J., and Heintz, N. 2003. Beclin 1, an autophagy gene essential for early embryonic development, is a haploinsufficient tumor suppressor. Proc. Nat1. Acad. Sci. 100: 15077-15082.

Zong, W.-X., Ditsworth, D., Bauer, D.E., Wang, Z.-Q., and Thompson, C.B. 2004. Alkylating DNA damage stimulates a regulated form of necrotic cell death. Genes \& Dev. (this issue). 


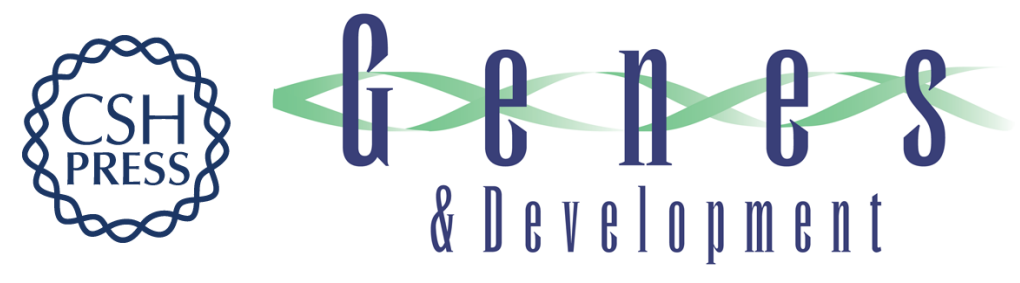

\section{Exploiting different ways to die}

Deirdre A. Nelson and Eileen White

Genes Dev. 2004, 18:

Access the most recent version at doi:10.1101/gad.1212404

References This article cites 21 articles, 5 of which can be accessed free at: http://genesdev.cshlp.org/content/18/11/1223.full.html\#ref-list-1

License

Email Alerting Receive free email alerts when new articles cite this article - sign up in the box at the top Service right corner of the article or click here.

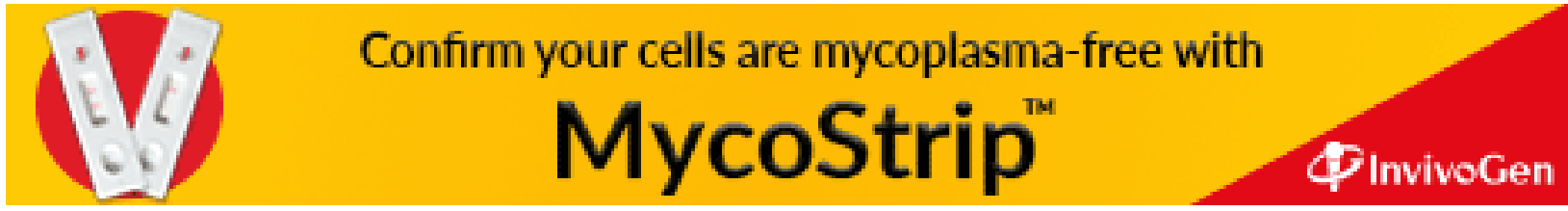

